\title{
Coordination games over Collision Channels
}

\author{
Eitan Altman *, Tania Jiménez ${ }^{\dagger}$, Nelson Vicuña ${ }^{\dagger}$, Richard Márquez ${ }^{\ddagger}$ \\ * INRIA, Centre Sophia-Antipolis, 2004 Route des Lucioles, 06902 Sophia-Antipolis Cedex, France \\ $\dagger$ Univesité d'Avignon, 339, Chemin des Meinajaries, Agroparc BP 1228, 84911 Avignon Cedex 9, France \\ $\ddagger$ Facultad de Ingenería, Universidad de Los Andes, Mérida 5101, Venezuela
}

\begin{abstract}
We consider competition between non-cooperative mobiles over several independent collision multiple-access channels or more generally, over interference channels. A mobile selects one of the channels for transmission; if the same channel is chosen simultaneously by more than a single mobile then a collision occurs and the colliding packets are lost. Policies based on assigning each independent channel to another mobile turn out to constitute efficient equilibria. But they request some synchronization and coordination which may not be possible. We identify other mixed equilibria that do not request synchronization but exhibit innefficiency. Moreover, we show that a Braess type paradox occurs in which the availability of more resources results in poorer performance to all mobiles. We extend the model to investigate the influence of capture phenomena.
\end{abstract}

\section{INTRODUCTION}

A large effort has been dedicated in recent years to the study of the multiple access channel [2], [15], [17], the interference channel [27], [18], [22], [25] and the relay channel [20] under game theoretic frameworks. Most of these references obtain a single equilibrium.

The goal of our work is to better understand the occurrence of multiple equilibria and their properties in some multiple access and in interference channels.

We start by describing a simple two players game in backlogged multiple access collision channels. Three equilibria are identified under symmetric conditions of which two are pure and one is a mixed equilibrium. The pure equilibria perform better than the mixed equilibrium but require coordination which is often not possible. We then obtain a similar structure in a more general setting of a non-symmetric interference channel with capture. We note that a similar structure has been obtained also in the context of non-cooperative routing in loss networks [1] and in a power control problem over an interference channel in [6].

We show that this type of games is a special case of the well studied class of coordination games [4], and the characterization of the equilibria is then a direct consequence. We further obtain properties of the equilibria that are specific to this problem. Numerical examples are introduced to illustrate our findings. The allow us to identify a Braess-type paradox [10] in which the availability of more resources results in poorer performance to all mobiles. We briefly discuss some stability issues and some extensions of the model.

The structure of the paper is as follows. After describing in the next section some central ideas through a simple motivating example, we present the general model and results in Section III. The stability of the equilibria is briefly discussed in Section IV. A numerical study is presented in Section V in which a Braess-type paradox is identified. Extensions to more than two mobiles are presented in Section VI followed by a concluding section.

\section{Simple Motivating Example}

Consider the following basic example. There are two mobiles $i=1,2$ and two independent channels $j=1,2$. Each mobile transmits at the same time one packet: Mobile $i$ transmits a packet over channel $i$ with probability $p_{i}$ and with probability $1-p_{i}$ over the other channel. A packet is successfully transmitted if it is the only one that uses the channel. Thus the transmission success probability of mobile $i$ is

$$
U_{i}(p)=p_{i} p_{j}+\left(1-p_{i}\right)\left(1-p_{j}\right), \quad j \neq i
$$

Mobile $i$ wishes to maximize the probability $U_{i}$ of successful transmission of its packet.

The policy that assigns a dedicated channel to each mobile (i.e. $p_{1}=p_{2}=1$ or $p_{1}=p_{2}=0$ ) is obviously optimal: it involves no collisions and the success probability is one. It is also a Nash equilibrium. However it requires coordination or synchronization in order to assign each channel to a different mobile.

The symmetric policy $p_{1}=p_{2}=0.5$ turns out to be an equilibrium; if mobile $i$ uses $p_{i}=0.5$ then no matter what $p_{j}$ mobile $j(i \neq j)$ chooses, it will have the same success probability of $1 / 2$. Thus no mobile can benefit by unilaterally deviating from $p=1 / 2$, so it is an equilibrium.

Note that if mobile $i$ had only one option, that of choosing channel $i$, then the innefficient equilibrium would not occur. This is a feature similar to the inefficiency we have in the prisoner's dilemma or in the Braess' paradox in which eliminating some options for the players can result in better performance to every one. Yet if we wanted to implement this idea in our context and create mobiles with only one channel, then we would face again a synchronization problem. If half of the mobiles have built in technology for accessing one channel and the other half can only access the other channel, then two randomly selected mobiles will still be using the same channel with probability half.

\section{THE MODEL AND MAIN RESULT}

Consider 2 mobiles and 2 base stations. The base stations use, each one, an independent channel (for example, each one uses another frequency). We shall assume that mobile $i$ has a good radio channel with base station $i$ and a bad one with 
station $j \neq i$. More precisely, let $h_{i j}$ be the gain between mobile $i$ and base station $j$.

Let $S I N R_{i}$ denote the Signal to Interference and Noise Ratio corresponding to the signal received from mobile $i$ at the base station to which it transmits. Each mobile has two pure strategies: $\gamma, \beta$ where $\gamma$ means transmitting on its good channel and $\beta$ on its bad one. Then

$$
S I N R_{1}(u)= \begin{cases}\frac{h_{11} P_{1}}{N_{o}} & \text { if } u=(\gamma, \gamma) \\ \frac{h_{12} P_{1}}{N_{o}} & \text { if } u=(\beta, \beta) \\ \frac{h_{11} P_{1}}{N_{o}+h_{21} P_{2}} & \text { if } u=(\gamma, \beta) \\ \frac{h_{12} P_{1}}{N_{o}+h_{22} P_{2}} & \text { if } u=(\beta, \gamma) \\ \frac{h_{22} P_{2}}{N_{o}} & \text { if } u=(\gamma, \gamma) \\ \frac{h_{21} P_{2}}{N_{o}} & \text { if } u=(\beta, \beta) \\ \frac{h_{22} P_{2}}{N_{o}+h_{12} P_{1}} & \text { if } u=(\beta, \gamma) \\ \frac{h_{21} P_{2}}{N_{o}+h_{11} P_{1}} & \text { if } u=(\gamma, \beta)\end{cases}
$$

Here $N_{o}$ is the thermal noise at each base station and $P_{i}$ is the fixed transmission power of mobile $i$.

Under many modulation schemes the probability of a successful transmission of a packet is known to be a monotone increasing function of the $S I N R$ [24]. We thus assume that mobile $i$ has a success probability given by $f_{i}\left(S I N R_{i}\right)$. Define

$$
\begin{aligned}
& A:=f_{1}\left(\frac{h_{11} P_{1}}{N_{o}},\right) \quad B:=f_{1}\left(\frac{h_{11} P_{1}}{N_{o}+h_{21} P_{2}}\right) \\
& C:=f_{1}\left(\frac{h_{12} P_{1}}{N_{o}+h_{22} P_{2}}\right) \quad D:=f_{1}\left(\frac{h_{12} P_{1}}{N_{o}}\right) . \\
& a:=f_{2}\left(\frac{h_{22} P_{2}}{N_{o}},\right) \quad b:=f_{2}\left(\frac{h_{22} P_{2}}{N_{o}+h_{12} P_{1}}\right) \\
& c:=f_{2}\left(\frac{h_{21} P_{2}}{N_{o}+h_{11} P_{1}}\right) \quad d:=f_{2}\left(\frac{h_{21} P_{2}}{N_{o}}\right) .
\end{aligned}
$$

The mobiles are thus faced with the following matrix game:

\begin{tabular}{|l|c|c|}
\hline & action $\gamma$ & action $\beta$ \\
\hline action $\gamma$ & $A, a$ & $B, c$ \\
\hline action $\beta$ & $C, b$ & $D, d$ \\
\hline
\end{tabular}

Theorem 3.1: There are exactly three equilibria; the two pure equilibria: $(\gamma, \gamma)$ and $(\beta, \beta)$, and a mixed one in which player 1 and 2 select $\gamma$ with probabilities:

$$
\begin{aligned}
& X^{*}=\frac{D-B}{A+D-B-C}, \quad Y^{*}=\frac{d-b}{a+d-b-c} . \\
& \text { Proof: }
\end{aligned}
$$
$c<a$. The game is thus a standard coordination game (see http://en.wikipedia.org/wiki/Coordination_game) [4] for which the result is well known.

The mixed equilibrium is characterized by the indifference property: when a mobile uses its mixed equilibrium policy then the other player is indifferent between $\gamma$ and $\beta$.

The utility of mobile 1 and 2 at the mixed equilibrium are given by

$$
U_{1}^{*}=A Y^{*}+B\left(1-Y^{*}\right), \quad U_{2}^{*}=a X^{*}+b\left(1-X^{*}\right) .
$$

Consider now the symmetric case $(A=a, B=b, C=c, D=$ $d)$. Then we get at the mixed equilibrium:

$$
U^{*}=\frac{(a-c)(d-c)+c(a+d-b-c)}{a+d-b-c}=\frac{a d-c b}{a+d-b-c}
$$

\section{Stability}

For any policy $Y$ for mobile $X$, the utility of mobile 1 is linear in the probability $X$ it selects $\gamma$. Only for $Y^{*}$ this utility does not depend on $X$, and thus for any other $Y$ the optimal response of mobile 1 is either one or zero. Thus a small deviation of the second mobile from $Y^{*}$ results in a big jump in the best response of the first mobile. But this is true for any mixed equilibrium in a matrix game.

We note however that the equilibrium is stable in the following sense: for any $\epsilon>0$, if mobile 2 deviates from $Y^{*}$ to a point $Y$ sufficiently close to $Y^{*}$ then $\left(X^{*}, Y\right)$ is an $\epsilon$-Nash equilibrium (i.e. a mobile cannot gain more than $\epsilon$ by deviating unilaterally).

A more formal notion of stability of an equilibrium comes from evolutionary games in which there are many pairwise interactions between individuals of an infinite population. The mixed strategies can be interpreted as the fraction of the population that uses the different pure policies. A multi-policy $\left(Z^{*}\right)$ is said to be an Evolutionary Stable Strategy (ESS) if it is robust against the deviation of a whole (sufficiently small) fraction of individuals. This is known to be equivalent (in the symmetric case) to the requirement that one of the following holds, see [26, Proposition 2.1]. For each $Y$, either

$$
U_{1}\left(Y, Z^{*}\right)<U_{1}\left(Z^{*}, Z^{*}\right)
$$

or

$$
U_{1}\left(Y, Z^{*}\right)=U_{1}\left(Z^{*}, Z^{*}\right) \text { and } U_{1}(Y, Y)<U_{1}\left(Y, Z^{*}\right)
$$

From the discussion above it can be seen that the mixed equilibrium $Z^{*}$ is not an ESS. Indeed, (1) does not hold and in fact for all $Y$ we have at the mixed equilibrium $U_{1}\left(Y, Z^{*}\right)=U_{1}\left(Z^{*}, Z^{*}\right)$. Moreover, when $Y$ corresponds to a pure strategy (in which each mobile is connected to another base station) then we have $U_{1}(Y, Y)>U_{1}\left(Y, Z^{*}\right)$ and then (2) clearly does not hold. 


\section{Numerical StUdy AND THE BRAESS' PARADOX}

As examples of success probability as a function of the SINR, we have the following expressions for the bit error probability as a function of the modulation [7] (numerical examples based on these formulas can be found in [3], [5], [7]):

$$
p_{e}(S I N R)=\left\{\begin{array}{lr}
\frac{1}{2} \operatorname{erfc}(\sqrt{\kappa \cdot S I N R}) & \text { for GMSK } \\
\frac{1}{2} \exp (-S I N R) & \text { for DBPSK } \\
\frac{1}{2} \exp \left(-\frac{1}{2} \cdot S I N R\right) & \text { for GFSK } \\
\frac{1}{2} \operatorname{erfc}(\sqrt{S I N R}) & \text { for 16-QAM } \\
\frac{3}{8} \operatorname{erfc}\left(\sqrt{\frac{2}{5} \cdot S I N R}\right) & \\
\frac{7}{32} \operatorname{erfc}\left(\sqrt{\frac{4}{21} \cdot S I N R}\right) & \text { for 64-QAM }
\end{array}\right.
$$

where $\kappa$ is a constant (that depends on the amount of redundancy in the coding and on the frequency band). In the absence of redundancy this gives the following expression for $f$ of a packet of $N$ bits provided that the bit loss process is independent

$$
f(S I N R)=\left(1-p_{e}(S I N R)\right)^{N}
$$

We next study a numerical example and examine the impact of the gain $h_{i j}$ on the utility. We use the following values of the parameters to calculate $U^{*}: h_{11}=h_{22}=10 d B, \kappa=0.68$, $P_{1}=P_{2}=0.6 \mathrm{Watt}, N_{0}=-174 d B$ and $N=60$.

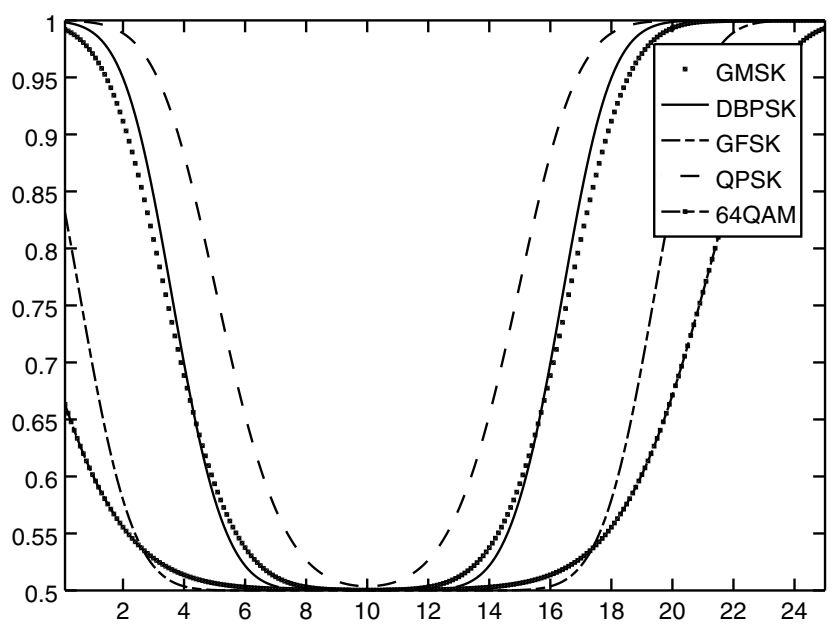

Fig. 1. Utility (vertical axis) at the mixed equilibrium with different values of the gain $h_{i j}$ in $\mathrm{dB}$ (horizontal axis)

We can see in Figure 1 that $U^{*}$ decreases when $h_{12}=h_{21}$ increase until they are equal to $h_{11}=h_{22}$. This behavior which occurs all along the interval, $\left(0, h_{11}=h_{22}\right)$, is known as a Braess-type Paradox.
This paradox is said to occur in a network whenever adding a link or adding capacity to a link causes delays of all users to increase; in an economic context in which users pay the service provider, this may further cause a decrease in the revenues of the provider. This problem was identified by Braess [10] in the transportation context, and has become known as the Braess' paradox. See also [14], [23]. The Braess' paradox has been studied as well in the context of queuing networks [9], [11], [12], [13], [16].

Remark 5.1: A behavior similar to the one we obtain in this access game over independent channels has been reported in [6] that studies an access game over independent interfering channels. There too, three equilibria have been identified in the case of two mobiles.

In our case, increasing the gains $h_{i j}$ (which can be done by using stronger antennas) can be considered as increasing the link capacity. As observed, a Braess' paradox indeed occurs.

\section{MORE THAN TWO MOBILES}

We return to the simple model of the Introduction extended to the case where there are $K$ mobiles and $K$ independent channels to some common base station. (The results are the same if we assume instead that each channel leads to another base station or access point). Note: access based on a random choice of independent collision channels can be found in satellite systems (see e.g. the SPADE Demand Assigned Multiple Access described in [21]) and in WiMAX.

We assume that if more than one transmission occurs simultaneously on a given channel then all packets involved are lost. Let $p=\left(p_{1}, \ldots, p_{K}\right)$ be a vector of probabilities. We interpret $p$ as a policy vector in which player $i$ transmits with probability $p_{i}$ over channel $i$ and with probability $\left(1-p_{i}\right) /(K-1)$ over a channel uniformly distributed among the remaining ones.

\section{A. The price of non-coordination}

It is easy to show that $p=\frac{1}{K}(1,1, \ldots, 1,1)$ is an equilibrium. It is a symmetric one which requires no coordination between the mobiles. We call this policy the non-coordinated equilibrium of degree $K$. The success probability of each mobile is

$$
U^{*}=\frac{1}{K}\left(1-\frac{1}{K}\right)^{K-1}
$$

The global utility is then

$$
G(K):=K U^{*}=\left(1-\frac{1}{K}\right)^{K-1}
$$

Note that this converges to $1 / e$ just as in the classical slotted ALOHA [19]!

The best equilibrium is obtained with $p=(1,1, \ldots, 1)$ for which the success probability of each mobile is 1 ; the global utility is then $K$. We call it the fully coordinated equilibrium. In order to implement it we need indeed to provide a mechanism enabling each mobile to choose a distinct channel. 
There are many other equilibria that we shall identify later, but the two we mentioned provide respectively the worst and the best throughput for each mobile.

The price of non-coordination which we define as the ratio between the non-coordinated equilibrium and the fully coordinated one is given by

$$
\operatorname{Pon} C(K)=\frac{1}{K}\left(1-\frac{1}{K}\right)^{K-1}
$$

Note that as $K \rightarrow \infty$ we get that PonC tends to zero and moreover

$$
\lim _{K \rightarrow \infty} K \operatorname{Pon} C(K)=1 / e
$$

\section{B. On the number of equilibria}

We show next that the number of equilibria grows exponentially with $K$. Let $m$ be some integer satisfying $1 \leq m \leq K$ and let $\widehat{K}=\left\{K_{1}, \ldots, K_{m}\right\}$ be a partition of $K$. Then we can identify $\widehat{K}$ as any policy in which $K_{i}$ mobiles restrict to some given $K_{i}$ channels and implement there the non-coordinated equilibrium of order $K_{i}$, for each $i=1,2 \ldots, m$.

Thus any partition of the $K$ mobiles defines another class of equilibria. The number of such classes is easily seen to grow exponentially fast with $K$.

\section{Equilibrium selection and evolution}

A large part of the literature on coordination game deals with equilibrium selection, where the question is how to learn to use the good equilibrium among the two pure ones. An example of an access problem in mobile communications that can be handled with these tools is the following.

Consider a group of $K$ mobiles along with $K$ receivers (base stations). We consider light traffic conditions so that we can neglect the probability that more than two mobiles along with their receivers are at interference range of each other. Each receiver has one independent radio channel which is better than the others. We assume symmetry; when in range with two receivers, we assume that mobile $i$ has a good radio channel with one receiver (the $i$ th, with a gain denoted by $h_{\gamma}$ ) and a bad channel with the other receiver (a gain of $h_{\beta}<h_{\gamma}$ ). There is a finite population of mobiles. The game is played repeatedly so mobiles have the opportunity to update their strategies. It is assumed that the mobiles are not aware of the utilities of other players and the question is then how to select a strategy.

This problem can be analyzed using model studied in [8]. In [8], the simple clever myopic adjustment strategy is considered where a player evaluates its strategy by comparing the current expected payoff to the expected payoff if it were to switch strategy, given that the remaining players do not switch their strategies. Two variants of this adaptation rule are considered in [8], and convergence to one of the pure equilibria is established in the limit as the size of the population of players grows to infinity.

\section{CONCLUding REMARKS}

This paper identifies the well known coordination games structure in a class of wireless non-cooperative games over multiple access collision channels or interference channels. The equilibria are computed for these games and their properties are studied. Through a numerical example, we identify a Braess-type paradox similar to the one obtained in [6], [16]. An interesting question which we leave for future work is whether similar multi-equilibria structure arise in the case of state dependents models.

\section{Acknowledgement}

This work was supported by the French-Venezuelan ECOSNord grant V04M01.

\section{REFERENCES}

[1] E. Altman, R. El-Azouzi and V. Abramov, "Non-Cooperative Routing in Loss Networks" , Performance Evaluation, Vol 49, Issue 1-4, pp. 257 272, 2002.

[2] E. Altman, R. El-Azouzi and T. Jimenez, "Slotted Aloha as a stochastic game with partial information", COMNET, Volume 45, Issue 6, pp. 701713, 2004

[3] D. Barman, I. Matta, E. Altman, R. El Azouzi, "TCP Optimization through FEC, ARQ and Transmission Power Tradeoffs", 2nd international Conference on Wired/Wireless Internet Communications WWIC 2004 Frankfurt (Oder), Germany, February 05 - 07, 2004.

[4] Russell Cooper: Coordination Games, Cambridge University Press, 1998 (ISBN 0-521-57896-5).

[5] L. Galluccio, G. Morabito and S. Pallazzo, "An analytical study of tradeoff between transmission power and FEC for TCP optimization in wireless networks", Proceedings of IEEE INFOCOM, 2003

[6] E. Altman, V. Kamble and H. Kameda, "A Braess type paradox in interference channels", submitted.

[7] J. G. Proakis, Communication Systems Engineering, Prentice Hall International Editions, 1994.

[8] W. H. Sandholm and A. Pazner, "Evolution, population growth and history dependence", Games and Economic Behavior 22, 84-120, 1998.

[9] N. G. Beans, F. P. Kelly, and P. G. Taylor. Braess's paradox in a loss network. J. Appl. Prob., 34:155-159, 1997.

[10] D. Braess. Uber ein paradoxen der werkehrsplannung. Unternehmenforschung, 12:256-268, 1968.

[11] B. Calvert, W. Solomon, and I. Ziedins. Braess's paradox in a queueing network with state-dependent routing. J. Appl. Prob., 34:134-154, 1997.

[12] J. E. Cohen and C. Jeffries. Congestion resulting from increased capacity in single-server queueing networks. IEEE/ACM Trans. on Networking, 5(2):1220-1225, April 1997.

[13] J. E. Cohen and F. P. Kelly. A paradox of congestion in a queuing network. J. Appl. Prob., 27:730-734, 1990.

[14] S. Dafermos and A. Nagurney. On some traffic equilibrium theory paradoxes. Transportation Research B, 18:101-110, 1984.

[15] H. Inaltekin and S. Wicker, "A one-shot random access game for wireless networks", International Conference on Wireless Networks, Communications and Mobile Computing, 2005

[16] H. Kameda. How harmful the paradox can be in Braess/Cohen-KellyJeffries networks. In IEEE INFOCOM, New York, NY, USA, June 2002.

[17] R. J. La and V. Anantharam, "A game-theoretic look at the Gaussian multiaccess channel", DIMACS Workshop on Network Information Theory, Rutgers University, Piscataway, NJ, USA, March 2003.

[18] O. Popescu, C. Rose, D.C. Popescu, "Signal space partitioning versus simultaneous water filling for mutually interfering systems" GLOBECOM'04 Global Telecommunications Conference, 2004.

[19] Raphael Rom and Moshe Sidi, Multiple access protocols: performance and analysis, Springer-Verlag New York, Inc., 1990 (ISBN 0-387-972536).

[20] Y. E. Sagduyu and A. Ephremides, "A game theoretic look at a simple relay channel", Wireless Networks, Volume 12, Number 5, pp. 1545-560, oct. 2006

[21] M. Schwartz, Information, Transmission, Modulation and Noise, 3rd Edition, New York: McGraw-Hill, 
[22] O. Simeone, Y. Bar-Ness, "Game theoretic view on the interference channel with random access," IEEE DySPAN 2007, April 17-20, 2007, pp 13-21.

[23] M. J. Smith. In a road network, increasing delay locally can reduce delay globally. E. Altman, R. El-Azouzi and V. Abramov, "Non-Cooperative Routing in Loss Networks" , Performance Evaluation, Vol 49, Issue 1-4, pp. 257-272, 2002 Transportation Res., 12:419-422, 1978.

[24] Pablo Soldati, Cross-Layer Optimization of Wireless Multi-hop Networks, Royal Institute of Technology (KTH), 2007 (ISBN 978-7178-7118).

[25] Y. Su and M. van der Schaar, ”New perspective on multi-user power control games in interference channels", available at http://arxiv.org/ftp/arxiv/papers/0709/0709.3880.pdf

[26] J.W. Weibull. Evolutionary Game Theory. newblock Cambridge, MA: MIT Press, 1995

[27] Wei Yu and JM Cioffi, "Competitive equilibrium in the Gaussian interference channel", in Proc. of ISIT, p. 431, June 2000. 